\title{
Strates géologiques et structure de la mémoire au début du XIX ${ }^{\mathrm{e}}$ siècle
}

Memory Strata, Geology and Change of Historical Paradigm in France around 1830

\section{Paule Petitier}

\section{(2) OpenEdition}

\section{Journals}

Édition électronique

URL : https://journals.openedition.org/aes/2129

DOI : 10.4000/aes.2129

ISSN : 2258-093X

Éditeur

Laboratoire LISAA

Référence électronique

Paule Petitier, "Strates géologiques et structure de la mémoire au début du xIx ${ }^{\mathrm{e}}$ siècle ", Arts et Savoirs [En ligne], 12 | 2019, mis en ligne le 24 février 2020, consulté le 21 septembre 2021. URL : http:// journals.openedition.org/aes/2129; DOI : https://doi.org/10.4000/aes.2129

Ce document a été généré automatiquement le 21 septembre 2021.

Centre de recherche LISAA (Littératures SAvoirs et Arts) 


\title{
Strates géologiques et structure de la mémoire au début du XIX ${ }^{\mathrm{e}}$ siècle
}

\author{
Memory Strata, Geology and Change of Historical Paradigm in France around \\ 1830
}

Paule Petitier

1 L'« ouverture du temps abyssal de la géologie autour de 1830 » constitue, selon Marcel Gauchet l'un "des grands événements de pensée $»^{1} \mathrm{du}$ XIX ${ }^{e}$ siècle. Dans la première moitié du XIXe siècle, le développement des études stratigraphiques - lié aux besoins de l'industrie en charbon et en minerais - répand y compris visuellement, par les frappantes représentations en coupes des multiples couches superposées composant un paysage, l'idée de ce temps profond, de ce passé littéralement entassé sous nos pieds. Les conséquences en sont importantes, comme le suggère la brève allusion de $\mathrm{M}$. Gauchet. Le « sombre abîme du temps » (Buffon) qui s'enfonce dans le passé bien avant l'apparition de l'homme, relativise extraordinairement l'histoire de celui-ci : «l'histoire humaine, désormais minoritaire [n'est plus $\mathrm{qu}^{\prime}$ ] une pellicule de connaissances relatives flottant à la surface d'un abysse ignoré $»^{2}$. Autre blessure narcissique pour l'humanité, s'ajoutant à celles qu'identifie Freud (Copernic, Darwin et Freud lui-même). La deuxième transformation importante consiste en ce que la géologie invite à penser la permanence du passé dans le présent. Le passé n'a pas disparu, il est là, invisible, enfoui mais possible à exhumer, et constitue même, matériellement, le présent où nous vivons.

2 Comme elle touche le rapport au temps, il n'est pas étonnant que cette révolution de pensée entre en résonnance avec la manière dont l'homme conçoit son intériorité. On constate en effet à travers de nombreux exemples littéraires une très rapide transposition du temps profond de la géologie à celui de la psychologie. Les phénomènes géologiques deviennent l'une des figurations privilégiées des mystérieuses profondeurs du moi. Pensons à la grotte du Schreckenstein dans laquelle le héros sandien Albert de Rudoldstadt se réfugie lors de ses graves crises de mélancolie, une grotte dans laquelle persiste le souvenir de la révolte hussite, censuré par l'histoire 
officielle (Consuelo). Ou encore, pour en rester aux exemples les plus évidents, à la grotte sous-marine dans laquelle Giliatt rencontre la pieuvre et dont les nervures minérales rappellent un cerveau (Les Travailleurs de la mer).

3 La représentation de la mémoire comme composée de strates est devenue banale aujourd'hui. La naturalisation de cette image s'explique certainement par la diffusion d'un modèle du psychisme théorisé par Freud, illustré par exemple dans une lettre à Fliess :

Tu sais que dans mes travaux, je pars de l'hypothèse que notre mécanisme psychique s'est établi par un processus de stratification: les matériaux présents sous forme de traces mnémoniques sont de temps en temps remaniés selon les circonstances nouvelles. ${ }^{3}$

La phrase de Proust, dans Albertine disparue, soulignant le caractère composite du « moi » n'est sans doute pas étrangère, elle non plus, à la fortune de cette métaphore :

Notre moi est fait de la superposition de nos états successifs. Mais cette superposition n'est pas immuable comme la stratification d'une montagne. Perpétuellement des soulèvements font affleurer à la surface des couches anciennes. ${ }^{4}$

Cependant c'est aux occurrences plus anciennes que je voudrais remonter, tentant ainsi de revenir au sens de cette métaphore avant qu'il ne se soit pour ainsi dire figé dans son périmètre actuel, modelé par les conceptions freudiennes. Cela me conduit à m'intéresser à celui qui me paraît le véritable «inventeur » de cette représentation du temps et de la mémoire marquée par le modèle des strates géologiques, le Chateaubriand des Mémoires d'outre-tombe.

\section{Le temps paradigmatique des Mémoires d'outre-tombe}

Certaines des œuvres antérieures au monument autobiographique, l'Essai sur les Révolutions et le Génie du christianisme, attestent que Chateaubriand connaissait les strates géologiques, même s'il se réfère à des interprétations à la fois datées et apologétiques : les " couches parallèles et horizontales des sols $\|^{5}$ sont prises à témoin du Déluge dans l'Essai sur les Révolutions, elles apparaissent dans le Génie comme des marques de l'âge de la Terre comparables aux anneaux de croissance des arbres ou de certains animaux (cornes des cerfs, dents du cheval, etc.) ${ }^{6}$.

7 Dans les Mémoires d'outre-tombe, cependant, l'allusion aux couches géologiques prend un plus grand rôle, puisqu'elle sert à caractériser ce qui constitue la matière même de l'œuvre, la mémoire. Elle remplit de ce fait une fonction métadiscursive, en particulier dans la IV ${ }^{e}$ partie, lorsqu'elle entre en résonance avec les caractéristiques d'une écriture largement fondée sur les redites et les parallélismes de la vie personnelle et de l'histoire.

Nos ans et nos souvenirs sont étendus en couches régulières et parallèles, à différentes profondeurs de notre vie, déposés par les flots du temps qui passent successivement sur nous.?

Dans la quatrième partie des Mémoires d'outre-tombe le fonctionnement paradigmatique de la mémoire prend le pas sur l'enchaînement séquentiel. Comme le rappelle JeanClaude Berchet, cette partie n'a pas été écrite en dernier lieu, mais « [s]a rédaction a, au contraire, accompagné celle des précédentes, si bien qu'elle a la particularité de réfléchir, comme dans un miroir, toutes les autres $»^{8}$. S'ouvrant « au sortir du fracas des 
trois journées» (p. 29) révolutionnaires qui ont en quelque sorte brisé l'histoire, la quatrième partie contrevient de plusieurs façons à la continuité du récit rétrospectif et adopte un principe de juxtaposition, tant par la diversité des formes (journal, lettres, citations) que par la généralisation de l'asyndète et de la rupture thématique (que Chateaubriand appelle "arabesque »). Le temps d'après 1830, cahoteux et fragmenté, tisse moins de liens entre un moment et celui qui le suit qu'entre l'instant présent et un passé lointain. Pour Jean-Claude Berchet la quatrième partie tisse des rapports avec la première; c'est même son sens profond que de faire revivre sur un autre mode le thème de l'exil et des chimères. Jeté sur les routes de l'Europe au service des Bourbons déchus, Chateaubriand croise et recroise ses itinéraires précédents, ce qui l'invite à une curieuse vision stéréoscopique superposant des images d'époques différentes. La répétition joue même à court terme : l'ambassadeur de la duchesse de Berry se rend deux fois à Prague, passe et repasse dans la bourgade bavaroise de Waldmünchen. L'enchaînement immédiat des faits ne semble plus vraiment essentiel ; l'intérêt du récit se recentre sur des plongées incessantes vers les souvenirs rattachés à un même lieu. On comprend dès lors que la métaphore des couches géologiques s'applique particulièrement à la mémoire dans cette partie de l'œuvre.

9 De façon répétée un lieu évoque deux états (du monde et de l'âme) différents, souvent antithétiques, séparés par un laps de temps variable, et dont l'évocation prend régulièrement la forme d'un parallélisme. C'est le cas par exemple, d'un passage près du lac Majeur :

Deux fois j'avais rencontré ce lac, une fois en me rendant au congrès de Vérone, une autre fois en allant en ambassade à Rome. Je le contemplais alors au soleil, dans le chemin des prospérités; je l'entrevoyais à présent la nuit, du bord opposé, sur la route de l'infortune. Entre mes voyages, séparés seulement de quelques années, il y avait de moins une monarchie de quatorze siècles. (p. 161)

Les souvenirs appartenant à des couches différentes font ainsi apparaître des dissemblances, plus encore des ruptures. En géologie, les partisans du catastrophisme pensent que la succession des strates géologiques correspond aux changements du climat, du niveau des eaux, de la flore et de la faune, et révèle des "créations » différentes, séparées par des bouleversements violents, des révolutions qui renouvellent la face du globe. De même, le mémorialiste éprouve, en confrontant le présent aux couches anciennes de sa mémoire, l'engloutissement de mondes entiers ( "une monarchie de quatorze siècles »). Dans l'histoire comme dans la nature, les révolutions produisent des strates dissemblables avec leurs faunes spécifiques:

De temps en temps, la Révolution nous envoyait des émigrés d'une espèce et d'une opinion nouvelles; il se formait diverses couches d'exilés : la terre renferme des lits de sable ou d'argile, déposés par les flots du déluge. ${ }^{9}$

11 Cette remarque introduit l'arrivée à Londres de Fontanes, présenté justement moins comme un individu que comme le représentant d'une époque, révolue au moment où écrit Chateaubriand. Fontanes apparaît comme l'incarnation d'un classicisme tardif dont le trait distinctif serait la mélancolie. Son portrait souligne à la fois les rapports étroits qui le lient à son temps (il porte "l'empreinte de l'époque où il a vécu ", aussi bien dans sa prose que dans ses vers) et le fossé qui le sépare, pour ainsi dire ontologiquement, de la génération de Chateaubriand.

[...] il ne pouvait ramener ces productions [Atala, René] aux règles communes de la critique, mais il sentait qu'il entrait dans un monde nouveau; il voyait une nature nouvelle; il comprenait une langue qu'il ne parlait pas. (ibid.) 
12 L'image des couches géologiques, à l'ouverture du chapitre, est intimement liée au développement qui suit. Elle est cohérente avec une vision du passé qui postule à la fois la consistance des époques (elles forment des touts ayant leur caractère propre) et la rupture entre les différentes époques. Elle conduit donc à penser le passé selon le modèle de la «coupe ", ou si l'on préfère de comparaisons entre points temporels distants, plutôt qu'en termes d'enchaînement continu.

\section{La durée plutôt que l'événement}

13 1830, par l'effet de répétition et de confirmation du caractère irréversible de la Révolution française, a sans doute modifié la vision que Chateaubriand avait de l'histoire et des processus historiques : «Le mouvement de Juillet, écrit-il dans "Ce que sera la révolution de Juillet", ne tient point à la politique proprement dite ; il tient à la révolution sociale qui agit sans cesse. $»^{10}$

La secousse historique qui détourne Chateaubriand de la carrière politique, le rend à un certain exil, et réenclenche l'écriture de ses Mémoires. Elle le conduit aussi à une relativisation de l'événement. Non seulement parce que les trois journées de Juillet n'ont été qu'un événement de proportions mesquines exploité par des politiques de piètre envergure aboutissant à une " quasi-légitimité » (tout est dans le " quasi »), mais parce qu'elles n'ont fait qu'entériner une transformation déjà accomplie et accomplie par d'autres voies, celles de "la révolution sociale qui agit sans cesse ". ${ }^{11}$ (c'est moi qui souligne). Pourtant, c'est le paradoxe que développe ce chapitre, la Révolution de Juillet, petite en elle-même, a soudain révélé l'épuisement de la France monarchique, fait apparaître celle-ci comme définitivement fossile.

À peu près au moment où Chateaubriand décide de reprendre ses Mémoires commencés et d'en faire sa grande œuvre, le géologue anglais Charles Lyell fait paraitre les trois volumes de ses Principes de la géologie (1830-1833). Cet ouvrage lu bien au-delà du cercle des scientifiques (15000 exemplaires vendus et 11 éditions jusqu'en 1872) s'opposait à la théorie catastrophiste de Cuvier et affirmait que pour expliquer les phénomènes naturels, on n'avait pas besoin de recourir à d'autres causes qu'à celles que l'on voyait à l'œuvre dans le moment présent. Son "uniformitarisme" proposait donc de faire l'économie des bouleversements violents et rapides de la surface du globe, causes selon Cuvier de renouvellements complets de la faune et de la flore. L'action imperceptible mais indéfiniment répétée des agents actuels (érosion, sédimentation, lents soulèvements de terrain) suffisait à expliquer, si l'on admettait son action sur des durées suffisamment longues, les transformations à terme les plus monumentales. À sa suite, Darwin allait bientôt expliquer de la même façon l'évolution des espèces, processus lent qui se poursuit à chaque instant sous nos yeux. Je ne veux d'aucune manière suggérer que Chateaubriand aurait lu Lyell, dont les théories ne s'imposeront que tardivement en France. Vers 1830 la géologie française est encore dominée par la théorie des révolutions, que prolonge l'œuvre d'Élie de Beaumont, consacrée à la formation des systèmes de montagnes. Comme on l'a vu, c'est plutôt a priori à cette vision d'une histoire du globe marquée par une ou des catastrophes (dont le Déluge biblique fournit le paradigme) que se rattachent directement les références géologiques de Chateaubriand. Et pourtant, tout se passe comme si, dans ce tournant de 1830 qui en Angleterre voit, sous l'influence de l'uniformitarisme, se modifier la compréhension du passé en même temps que s'étend de manière extraordinaire la durée des temps 
géologiques, il se produisait en France une révolution de pensée comparable dans le domaine de l'histoire humaine. L'œuvre de Chateaubriand nous semble en porter la marque. «Il n'est révolution si prodigieuse, écrit encore Chateaubriand à propos de Juillet, qui, décrite de minute en minute, ne se trouvât réduite aux plus petites proportions. $»^{12}$

La révolution de Juillet oblige à un nouveau rapport à l'histoire. Faite par le peuple (même si elle lui a été confisquée), sans meneurs dira Michelet ${ }^{13}$, elle oblige à reconnaître un acteur historique multiple et anonyme. Observée en temps réel (temps qui constitue non seulement un point de vue mais aussi un rythme - « de minute en minute »), cette révolution de trois jours révèle que l'histoire ne s'accomplit pas dans une autre temporalité que celle de tous les jours. Elle prive l'Histoire de sa grande « $\mathrm{h}$ ", de ses héros, de ses actes d'emblée " historiques ». Comprenons bien qu'il ne s'agit pas de dénier l'histoire, les changements profonds qui s'y produisent mais de dissocier son mode d'effectuation (les causes habituelles toujours agissantes) des résultats que l'on perçoit après coup et à long terme (chutes des empires, dissemblances des générations, transformation des valeurs), résultats dont la vision à distance met puissamment en relief la grandeur. D'un côté le travail du temps, toujours présent sous nos yeux, de l'autre, l'histoire comme cumulation de ces résultats sur un temps long donnant à voir des changements massifs. Michelet reprochant à Victor Hugo une représentation trop dramatique de l'histoire, et ses sauts "de couleur en couleur, de montagne en montagne ", estime que l'historien doit au contraire "suivre les ondulations de la vie " ${ }^{14}$. Il faut désormais être chroniqueur, connaître intimement le travail du temps au jour le jour pour saisir la fabrique de l'histoire, et être capable d'embrasser rétrospectivement la très longue durée afin de saisir les résultats de l'histoire. On ne contemple la grandeur de l'histoire qu'avec la conscience du recul, de la mise en perspective sur laquelle repose cette grandeur. On ne peut saisir l'élaboration de l'histoire dans la trame même d'une vie humaine et apercevoir ses conséquences sans devoir changer d'échelle.

17 La promotion du temps de l'existence comme laboratoire de l'histoire permet de comprendre le nouveau statut du «moi » du mémorialiste. Alors qu'auparavant le mémorialiste, autorisé par sa position privilégiée de témoin (par sa proximité du pouvoir), déroulait le récit des événements plus ou moins importants auxquels sa vie avait été entremêlée, le mémorialiste postrévolutionnaire, lui, raconte sa propre vie à la lumière de laquelle il peut saisir l'histoire - qu'il occupe une position centrale ou périphérique par rapport au pouvoir. L'écriture à la première personne devient un instrument pour capter l'histoire. De deux façons : premièrement parce que le temps vécu n'est pas d'une autre nature que le temps de l'histoire - l'histoire, on l'a compris, ne se déroulant pas dans une temporalité autre, plus noble, voire transcendante. Deuxièmement parce que l'écoulement imperceptible du temps subjectif et individuel ménage de brusques prises de conscience, de brutales ouvertures sur un temps qui a désormais l'allure de l'histoire : «Ah ! dans trois mois j'aurai cinquante ans; est-il bien possible ? 1783, 93, $1803:$ je suis tout le compte sur mes doigts... et 1833 : cinquante. Est-il bien possible? $»^{15}$

18 Tant soit peu que la vie s'allonge, l'existence individuelle fournit le modèle de la conversion du temps vécu en histoire, au moment où l'accumulation des années produit du temps qui s'objective douloureusement, se transforme en chronologie. L'insistance de Chateaubriand sur sa longévité dans la quatrième partie des Mémoires 
d'outre-tombe n'est pas simple rappel du poids de la vieillesse ou coquetterie d'auteur. Il ne s'agit pas non plus d'avancer que l'âge doterait de plus de sagesse ou de savoir, ni même qu'il permettrait d'embrasser plus d'événements, mais la durée est une manière de se mettre au pas de l'histoire, de son travail continu, inscrit dans le cours commun des choses et pourtant frappant à terme. La durée de la vie devient un atout pour saisir par des jeux d'échelles ce jeu de perspective qu'est l'histoire.

\section{Jeux d'échelles}

Dans la quatrième partie des Mémoires d'outre-tombe, les strates de souvenirs incessamment réveillées par le voyage jouent le rôle d'appareil optique pour saisir les changements historiques sans pour autant se mystifier sur la façon dont ils se sont produits. Comme le montrait déjà la citation commentant les deux passages au bord du lac Majeur, la comparaison entre deux couches de mémoire personnelle fait mesurer à travers le temps vécu un autre intervalle, relevant lui de l'histoire: "Entre mes voyages, séparés seulement de quelques années, il y avait de moins une monarchie de quatorze siècles. » Cet emboîtement permet de combiner deux échelles ou deux rythmes autrement incompatibles : celui du travail du temps (égal à celui de la vie individuelle) et celui du sens de l'histoire (les grandes démarcations ou les grandes unités perceptibles seulement au-delà de leur terminus ad quem). Pour le formuler autrement, le passé simple de l'histoire (ce décrochement de certains événements par rapport au présent) ne peut être perçu qu'à travers le passé composé de l'expérience vécue (encore rattaché au présent).

L'attention aux redites de l'histoire personnelle rend Chateaubriand sensible aux répétitions de l'histoire, à la surimpression des événements dans les mêmes sites. Il remarque ainsi la réitération des batailles dans les mêmes lieux :

Entre Dilingen et Donauwerth, on traverse le champ de bataille de Blenheim. Les pas des armées de Moreau sur le même sol n'ont point effacé ceux des armées de Louis XIV; la défaite du grand roi domine dans la contrée les succès du grand empereur. [...] Rien ne fait mieux sentir la grandeur de Louis XIV que de trouver sa mémoire jusqu'au fond des ravines creusées par le torrent des victoires napoléoniennes. Les conquêtes de ce monarque ont laissé à notre pays des frontières qui nous gardent encore. L'écolier de Brienne, à qui la légitimité donna une épée, enferma un moment l'Europe dans son antichambre; mais elle en sortit : le petit-fils de Henri IV mit cette même Europe aux pieds de la France; elle y est restée. (p. 227)

La métaphore géologique revient, à travers l'image d'un sol érodé par le ruissellement d'un torrent, pour caractériser la trace profonde laissée dans les mémoires par les victoires napoléoniennes. Pourtant la marque en creux ne fait que mettre à nu un souvenir plus ancien, celui de Louis XIV. On pourrait s'en tenir au sens évident : l'éloge de la France monarchique, de sa gloire militaire supérieure à celle de l'Usurpateur. Mais au-delà du propos idéologique s'esquisse une autre histoire, voire une géopolitique. Le texte n'établit pas seulement une hiérarchie entre Louis XIV et Napoléon, mais aussi une continuité, celle des tentatives d'hégémonie européenne de la France dans le temps long. À moins que la surimpression ne révèle, peut-être fort pertinemment, un déclin: Louis XIV a été vaincu à Blenheim, mais il avait mis l'« Europe aux pieds de la France » tandis que les conquêtes napoléoniennes ont poussé 
l'Europe à se révolter contre le joug français. Napoléon marquerait en fin de compte l'apothéose et la butée de la "grande nation ».

On voit donc s'esquisser dans de tels passages d'autres " propositions » historiques que celles de l'histoire événementielle et politique. La métaphore des couches de la mémoire nous apparaît toujours liée à la question des échelles pour penser le passé, aux points de vue différents qu'elles donnent ainsi qu'à des suggestions d'interprétation historique fondées sur la longue durée. Revenons sur le contexte dans lequel affleure la phrase des Mémoires d'outre-tombe qui décrit le plus clairement la mémoire comme constituée de couches.

De retour de son premier voyage à Prague, Chateaubriand arrive à Metz.

Je fus frappé, en entrant à Metz, d'une chose que je n'avais pas remarquée en 1821;

les fortifications à la moderne enveloppent les fortifications à la gothique : Guise et

Vauban sont deux noms bien associés. (p. 361)

Nouveau phénomène de surimpression - de souvenirs de voyage, mais aussi de topographie urbaine. Ce que Chateaubriand n'avait pas remarqué avant la Révolution de 1830, c'est l'emboîtement des murailles c'est-à-dire de deux noms liés à la Monarchie, mais que tout apparemment opposait. D'un côté celui d'une famille princière, illustrée par la victoire de François au siège de Metz en 1552, mais aussi par l'opposition turbulente d'Henri au pouvoir royal pendant la Ligue. De l'autre, Vauban, issu de petite noblesse, exemple de ces hommes de talent qui se plièrent entièrement au service du grand roi. Après 1830, la topographie de Metz révèle en fin de compte la profonde parenté de ces deux noms renvoyant l'un et l'autre à la même période révolue de la vitalité monarchique. La surimpression des murailles appelle l'image des couches géologiques au paragraphe suivant :

Nos ans et nos souvenirs sont étendus en couches régulières et parallèles, à différentes profondeurs de notre vie, déposés par les flots du temps qui passe successivement sur nous. (ibid.)

Laquelle est aussitôt développée par un exemple mêlant une nouvelle fois souvenirs personnels et histoire :

C'est de Metz que sortit en 1792 la colonne engagée sous Thionville avec notre petit corps d'émigrés. J'arrive de mon pèlerinage à la retraite du prince banni que je servais dans son premier exil. Je lui donnai alors un peu de mon sang, je viens de pleurer auprès de lui ; à mon âge on n'a guère que des larmes. (ibid.)

Les deux passages par Metz mettent en relief l'opposition des âges (jeunesse/vieillesse) mais ce n'est que pour suggérer une identité par-delà l'antithèse. Même situation à travers les effets de symétrie, un prince déjà exilé, un combat déjà perdu. De sorte que, de la même façon que pour Guise et Vauban, l'intervalle temporel fait ressentir une continuité fondamentale, celle de la défaite de la monarchie jouée dès 1792 et confirmée par 1832. Un changement de paragraphe correspond à l'examen d'un nouvel intervalle temporel :

En $1821 \mathrm{M}$. de Tocqueville, beau-frère de mon frère, était préfet de la Moselle. Les arbres, gros comme des échalas que M. de Tocqueville plantait en 1820 à la porte de Metz, donnent maintenant de l'ombre. (ibid.)

Revenant aux beaux temps de la Restauration, Chateaubriand évoque un souvenir qui semble n'avoir aucun rapport avec les précédents. Cependant la « porte de Metz » est là pour rappeler Vauban et la vocation militaire de la cité lorraine. Mais planter des arbres est une activité pacifique, un détail qui rompt avec l'évocation de la gloire guerrière de la monarchie. Ces arbres ont d'ailleurs tranquillement continué leur 
croissance entre 1822 et 1832, nullement dérangés par la fin de la Restauration. Notons encore que le planteur, malgré son nom à particule, par sa fonction de préfet (création de l'Empire) et de préfet de la Moselle (nom d'un département créé par l'Assemblée Constituante) manifestait sous la Restauration même la continuité d'un ordre nouveau, destiné comme ses arbres à durer en dépit des révolutions politiques.

La référence aux strates de la mémoire correspond donc à des mises en perspective différentes de l'histoire, où le temps le plus banal (celui, par exemple, de la croissance des arbres) sert à faire entrevoir une vérité historique. Par ce jeu d'échelles, la vie individuelle permet de saisir des transformations historiques autres que celles de la chronologie traditionnelle fondée sur les événements et sans rapport avec les processus historiques réels.

L'analyse de la métaphore des strates de la mémoire chez Chateaubriand met en lumière l'importance du moment 1830 dans l'apparition d'un nouveau rapport au passé. 1830 est une révolution qui, paradoxalement, semble affaiblir l'idée d'une histoire événementielle, ponctuée de changements radicaux et rapides. La brièveté de la révolution de Juillet fait qu'elle apparaît comme la sanction de transformations à long terme, résultats de processus d'autant plus irréversibles qu'ils ont été multiformes et banals. La géologie accomplit à la même époque outre-Manche sa conversion à l'uniformitarisme et pose pour seuls facteurs de transformation ceux qu'il est loisible d'observer au présent. Il semble qu'en France on assiste à une conversion comparable dans le domaine de la pensée de l'histoire. On en trouve trace non seulement chez le grand mémorialiste qu'a été Chateaubriand, mais encore chez un romancier, Balzac, et chez un historien, Michelet - convergence qui me paraît probante. À la question "Qu'est-ce qui me constitue en être historique et en témoin de l'histoire? ?, chacun de ces trois auteurs apporte une réponse comparable. Pour le mémorialiste, ce n'est plus tant le fait d'avoir été témoin d'événements importants et d'en rapporter le déroulement, c'est la capacité de se servir de sa propre vie, du présent, pour observer comment le passé se transforme en histoire. Quant à Balzac, ses romans d'après 1830 ne rejoignent-ils pas cette voie? Ne s'agit-il pas désormais de saisir l'histoire dans le présent, de donner à voir sa fabrique réelle: la complexité des interactions sociales telle que le romancier l'a sous les yeux, et telle qu'elle explique les bouleversements profonds constatables ensuite à quelques décennies de distance ? Le présent est bien la meilleure façon de comprendre le passé, il permet de saisir l'histoire mieux que n'importe quel roman historique brodant une intrigue sur un canevas d'événements considérés comme décisifs seulement par un effet de perspective. Enfin, se tourner vers Michelet, c'est aussi constater que l'historien se double chez lui d'un diariste. Le Journal tenu quotidiennement n'enregistre pas les grands événements contemporains, mais l'existence, la régularité du travail, des fréquentations et même des fonctions physiques. Et pourtant le Journal est aussi le lieu où l'auteur observe les strates de sa vie intellectuelle et la construction de sa mémoire : l'enregistrement du présent constitue ainsi une modélisation de l'histoire. Dans son œuvre-manifeste de 1831, l'Introduction à l'histoire universelle, Michelet déclare avoir repensé l'histoire à la lumière de «l'éclair de Juillet » : en effet, cet éclair le conduit à plonger jusqu'aux racines de l'histoire humaine et à la présenter comme un très long voyage (sans événements) de l'Inde à la France. La fulgurance du présent débouche immédiatement sur une ouverture maximale du temps de l'histoire. 
1830 apparaît donc comme ce moment où le présent devient «le lieu dans lequel, véritablement, se joue la construction du passé $»^{16}$. Et ce que Laurent Olivier commentant la révolution épistémologique de Lyell en tire comme conclusion nous paraît au terme de cette réflexion parfaitement s'appliquer à nos auteurs de la monarchie de Juillet :

Du coup, l'histoire - comme connaissance du passé - en est transformée : elle n'est plus le récit de la succession des temps ou des périodes du passé : elle devient une discipline encore inédite qui observe le passé comme l'accumulation d'une mémoire toujours en construction. ${ }^{17}$

Figure

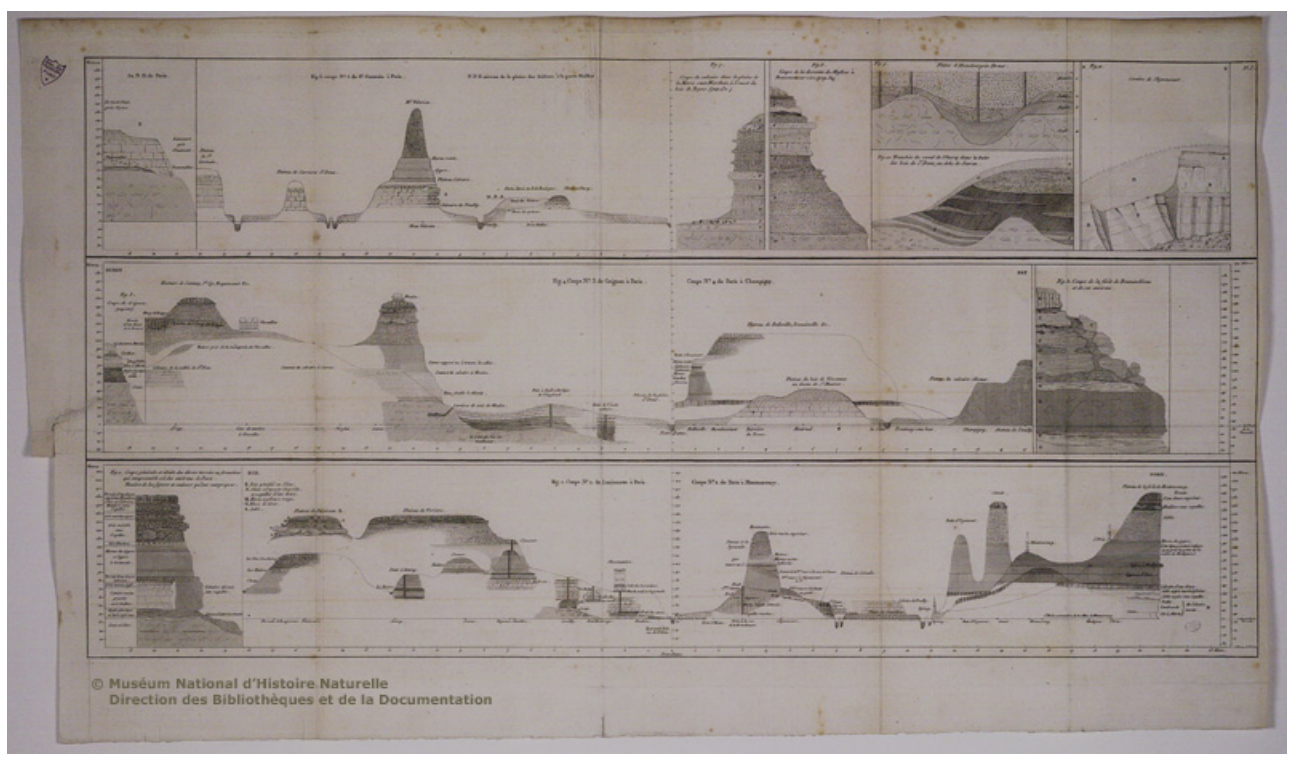

Georges Cuvier et Alexandre Brongniart, Planche extraite de l'Essai sur la géographie minéralogique des environs de Paris, Paris, Baudoin, 1811

(c) MNHN, Bibliothèque centrale, 15422.

Une version de cet article a été précédemment publiée dans Biological Time, Historical Time. Transfers and Transformations in 19th Century Literature, Niklas Bender et Gisèle Séginger (dir.), Brill | Rodopi, 2018, « Faux-Titre», p. 29-44, https://brill.com/view/title/39098.

\section{NOTES}

1. Marcel Gauchet, L'Inconscient cérébral, Paris, Seuil, 1992, coll. "La Librairie du Xxi ${ }^{\mathrm{e}}$ siècle », p. 69.

2. Laurent Olivier, Le sombre abîme du temps, Paris, Seuil, 2008, coll. « La couleur des idées », p. 32.

3. Sigmund Freud, Lettre $n^{\circ} 52$ du 6 décembre 1896, dans La Naissance de la psychanalyse, Paris, PUF, 2009, p. 153.

4. Marcel Proust, Albertine disparue, Paris, Gallimard, 1998 et 1992, Coll. « Folio », p. 126.

5. François-René de Chateaubriand, Essai historique sur les révolutions, dans Euvres complètes, Pourrat frères, 1834, t. I, p. 18. 
6. François-René de Chateaubriand, Génie du christianisme, I, IV, v., Paris, Garnier frères, 1828, p. 82.

7. François-René de Chateaubriand, Mémoires d'outre-tombe ${ }^{* * * *}$. Livres XXXIV à XLII, éd. J.-Cl. Berchet, Paris, Le Livre de poche, 1998, «Classiques Garnier»,p. 361. Les citations ultérieures renverront toutes à cette édition, les numéros de page étant indiqués entre parenthèses à la suite du texte.

8. J.-Cl. Berchet, « Notice des Livres XXXIV à XLII », éd. cit., p. 11.

9. Mémoires d'outre-tombe, livre XI, chapitre III.

10. Mémoires d'outre-tombe ${ }^{* * *}$, op. cit., , p. 560.

11. C'est moi qui souligne. Dans ce cas, il faut supprimer l'indication dans le texte.

12. Ibid., p. 558.

13. Jules Michelet, Introduction à l'histoire universelle, dans Euvres complètes, Paris, Flammarion, 1972 , t. II, p. 254-255.

14. Jules Michelet, «Dossier Méthode historique et enseignement ", Papiers Michelet (Cote A 3887), Bibliothèque historique de la Ville de Paris.

15. Stendhal, Vie d'Henry Brulard, Paris, Glénat, 1988, p. 18.

16. Laurent Olivier, op. cit., p. 205

17. Ibid. p. 210.

\section{RÉSUMÉS}

L'article étudie la métaphore des couches de la mémoire en relation avec les travaux des géologues du début du XIXe siècle sur l'étagement des couches du sous-sol. On observe, notamment dans les Mémoires d'outre-tombe de Chateaubriand, que l'appropriation de cette image par des écrivains et des historiens correspond à la Révolution de 1830. Cette transformation de la relation au temps et de la conception de la mémoire coïncide avec les thèses «actualistes » du géologue anglais Lyell. Il s'agit là sans doute non d'un rapport de cause à effet, mais de l'une de ces synchronies qui permettent d'esquisser une histoire des modèles de pensée.

The contribution analyses the metaphor of layers of memory in the precise moment of its creation, viz. at the beginning of the 19th century, in the works of Chateaubriand. Subsequently one can observe how its appropriation by the memorialist corresponds to a transformation of his conception of history during the Revolution of 1830 . This does not necessarily hint at a causal connection but rather at one of those synchronicities wich are the possible basis of a global history of the models of thought.

\section{INDEX}

Keywords : memory, geology, Chateaubriand, romantic History, 1830

Mots-clés : mémoire, géologie, Chateaubriand, Histoire romantique, 1830 
AUTEUR

PAULE PETITIER

Université de Paris. CÉRILAC (EA 1440) 